\title{
Prognostic value of the Quick Sepsis-related Organ Failure Assessment (qSOFA) score among critically ill medical and surgical patients with suspected infection in a resource- limited setting
}

\author{
L A Bishop, ${ }^{1} \mathrm{MB} \mathrm{ChB}$; D P K Wilson, ${ }^{1} \mathrm{MB} \mathrm{ChB}, \mathrm{FCP}(\mathrm{SA}), \mathrm{PhD} ; \mathbf{R}$ D Wise, ${ }^{2,3} \mathrm{MB}$ ChB, FCA (SA), Cert Crit Care, MMed; \\ S M Savarimuthu, ${ }^{4} \mathrm{MD}$; G L Anesi, ${ }^{5} \mathrm{MD}, \mathrm{MSCE}, \mathrm{MBE}$

\begin{abstract}
${ }^{1}$ Department of Internal Medicine, School of Clinical Medicine, University of KwaZulu-Natal, Pietermaritzburg, South Africa
${ }^{2}$ Discipline of Anaesthesiology and Critical Care, School of Clinical Medicine, University of KwaZulu-Natal, Durban, South Africa

${ }^{3}$ Adult Intensive Care Unit, John Radcliffe Hospital, Oxford University Hospitals NHS Foundation Trust, Oxford, United Kingdom

${ }^{4}$ Division of Pulmonary, Critical Care and Sleep Medicine, Yale School of Medicine, New Haven, Connecticut, USA

${ }^{5}$ Division of Pulmonary, Allergy, and Critical Care, University of Pennsylvania Perelman School of Medicine, Philadelphia, USA
\end{abstract}

Corresponding author: L A Bishop (leesabishop@gmail.com)

\begin{abstract}
Background. The Quick Sequential Organ Failure Assessment (qSOFA) score is a simple bedside tool validated outside of the intensive care unit (ICU) to identify patients with suspected infection who are at risk for poor outcomes.

Objectives. To assess qSOFA at the time of ICU referral as a mortality prognosticator in adult medical v. surgical patients with suspected infection admitted to an ICU in a resource-limited regional hospital in South Africa (SA).

Methods. We conducted a retrospective cohort study on adult medical or surgical patients that were admitted to an ICU in a resource-limited hospital in SA. We performed univariate and multivariable logistic regression and compared nested models using likelihood ratio test, and we calculated the area under the receiver operating characteristic curve (AUROC).

Results. We recruited a total of 1162 (medical $n=283$ and surgical $n=875$ ) participants in the study who were admitted to the ICU with suspected infection. qSOFA at the time of ICU referral was highly associated with but poorly discriminant of in-ICU mortality among medical (odds ratio (OR) 2.60, 95\% confidence interval (CI) $1.19-5.71 ; p=0.02$; AUROC 0.60; 95\% CI $0.53-0.67 ; p=0.02$ ) and surgical (OR 2.74; 95\% CI 1.73-4.36; $p<0.001$; AUROC $0.60 ; 95 \%$ CI $0.55-0.65 ; p=0.04)$ patients. qSOFA model performance was similar between medical and surgical subgroups $(p \geq 0.26)$. Addition of qSOFA to a baseline risk factor model including age, sex, and HIV status improved the model discrimination in both subgroups (medical AUROC 0.64; 95\% CI 0.56 - 0.71; $p=0.049$; surgical AUROC 0.69; $95 \%$ CI 0.64 - 0.74; $p<0.0001$ ).

Conclusion. qSOFA was highly associated with, but poorly discriminant for, poor outcomes among medical and surgical patients with suspected infection admitted to the ICU in a resource-limited setting. These findings suggest that qSOFA may be useful as a tool to identify patients at increased risk of mortality in these populations and in this context.
\end{abstract}

Keywords. intensive care; sepsis; qSOFA; adult; prognosis.

Afr J Thoracic Crit Care Med 2021;27(4):145-150. https://doi.org/10.7196/AJTCCM.2021.v27i4.158

Sepsis is a major global health burden with high morbidity and mortality. ${ }^{[1]}$ Approximately $30 \%$ of all intensive care unit (ICU) patients have or develop sepsis, ${ }^{[1]}$ defined as life-threatening organ dysfunction caused by a dysregulated host response to infection. ${ }^{[2]}$ More than a third of these patients do not survive their stay in hospital. ${ }^{[1]}$ The limited availability of ICU beds is a major problem in resource-limited settings. ${ }^{[3]}$ The ability to identify patients most likely to benefit from ICU admission may help ensure the optimal use of these facilities.

The quick Sequential Organ Failure Assessment (qSOFA) score is a bedside assessment tool introduced in the Sepsis-3 guidelines ${ }^{[2]}$ as a prognosticator of poor outcomes in patients with suspected infection, and validated outside but not inside of the ICU setting. ${ }^{[4]}$ The qSOFA includes three clinical parameters: $(i)$ blood pressure (threshold systolic blood pressure $\leq 100 \mathrm{mmHg}$ ); (ii) respiratory rate (threshold $\geq 22$ breaths per minute); and (iii) mentation (threshold Glasgow Coma Score $(\mathrm{GCS}) \leq 14$ ). Initial evaluation indicated that the presence of two or more qSOFA points is associated with a greater risk of death or prolonged ICU stay in developed countries. ${ }^{[2]}$

The qSOFA score is increasingly being used and studied, following its inclusion in the Sepsis-3 guidelines of 2016. Studies have shown mixed results in different patient populations and contexts. It appears that the qSOFA score may have low sensitivity but high specificity as a prognosticator of mortality. ${ }^{[5-12]}$ Age, sex and HIV status have been shown to independently predict mortality in patients with infection in the US and have been used in prior qSOFA validation studies in resource-limited settings. ${ }^{[13]}$ 
There is limited research on the prognostic value of qSOFA in resourcelimited settings - initial results from developing countries ${ }^{[13]}$ and African countries are encouraging. ${ }^{[14-17]} \mathrm{A}$ recent study in ICU patients with and without suspected infection showed that high qSOFA scores on admission were associated with an increased likelihood of in-ICU mortality in South Africa (SA). ${ }^{[18]}$ Further studies are required to assess the role of qSOFA score as a predictor of mortality in these settings.

The prognostic role of qSOFA in medical v. surgical cohorts is yet to be determined since studies to date have been conducted on mixed cohorts of patients. Therefore, studies are needed to assess qSOFA prognostic efficacy in these differing populations.

\section{Methods \\ Design}

We performed a single-centre, retrospective cohort study to evaluate the prognostic validity of the qSOFA score at the time of ICU referral in adult medical v. surgical ICU patients admitted with suspected infection. We utilised the Integrated Critical Care Electronic Database (ICED) ${ }^{[19]}$ at Edendale Hospital in Kwazulu-Natal Province, SA. Edendale Hospital is a regional-level public hospital with 900 beds that operates in a resource-constrained context. The ICU admits both surgical and medical patients. The ICU has capacity for nine patients but provides mechanical ventilatory support for a maximum of six patients due to staffing and equipment constraints. This study used an ICU outcomes analytic dataset assembled from the ICED database and used in prior published studies. ${ }^{[3,18]}$

ICED includes all referrals for ICU care at Edendale Hospital since 2014. Referrals come from the emergency department and wards, as well as from other district and community hospitals in the KwaZuluNatal Department of Health geographic catchment area. Data entry into ICED is integrated into the normal daily workflow and clinical documentation of the ICU, ensuring complete population capture.

Data entry categories include demographics, admitting diagnosis and subspecialty classification, referral information, clinical data, triage decision, and initial management plan including antibiotic prescription. Organ support, mortality, length of stay, and discharge destination are recorded at the end of the ICU stay. All entries are date and time stamped. Data are not collected after ICU discharge.

\section{Participants}

All adult patients ( $\geq 18$ years) admitted to the ICU from January 2014 to December 2018 with suspected infection were eligible for inclusion. 'Suspected infection' was defined as commencement or continuation of antibiotic therapy at the time of ICU admission. Patients accepted for admission but never physically transferred/ admitted to the ICU for any reason (i.e. due to bed unavailability or a change in clinical status) were excluded. Patients were identified as medical or surgical patients based on the referring specialty or as assigned by the ICU team, as recorded in the database.

\section{Exposure and outcomes}

The primary exposure was the qSOFA score at the time of ICU referral (i.e. before the commencement of ICU care). For primary analyses, qSOFA was treated as an ordinal variable with 0 - 1 (reference), 2 , and 3 points, due to the small number of study subjects with qSOFA score of 0 in this critical care population, and prior research showed a stepwise association with mortality. ${ }^{[18]}$ In sensitivity analyses, we treated qSOFA as dichotomous ( $<2 \mathrm{v} . \geq 2$ points) as per the Sepsis-3 guidelines. ${ }^{[2]}$ The primary outcome was in-ICU mortality, defined as death in the ICU or a palliative ICU discharge.

\section{Statistical analysis}

The baseline characteristics of the included patients were reported as mean (standard deviation (SD)) for continuous normally distributed variables; median and interquartile range (IQR) for data that were not normally distributed; and count (percent) for categorical and ordinal variables.

The proportion of patients with suspected infection who died in ICU in each category was calculated. To measure the association between qSOFA score and in-ICU mortality, we performed univariate and multivariable logistic regression analysis. We performed further analysis using a baseline risk model that included age, sex, and HIV status, which have been shown to be independently associated with mortality, to assess the additive prognostic benefit of qSOFA and allow comparison with related prior studies. ${ }^{[13]}$ We compared nested models (i.e. baseline v. baseline plus qSOFA) using likelihood ratio tests.

Since defining 'suspected infection' based on antibiotic therapy alone risks including some patients without acute infection (e.g. those receiving antibiotics for post-surgical prophylaxis, chronic infections, infection suppression, or anti-inflammatory indications) in a sensitivity analysis, we repeated our primary analysis including only patients for whom infection was the primary ICU admission diagnosis. This improved specificity at the expense of sensitivity (e.g. missing patients with a non-infectious indication for ICU admission listed primarily but also with acute infection).

Discrimination was assessed by calculating the area under the receiver operating characteristic curve (AUROC). Differences between AUROCs for medical and surgical patient subgroups were determined

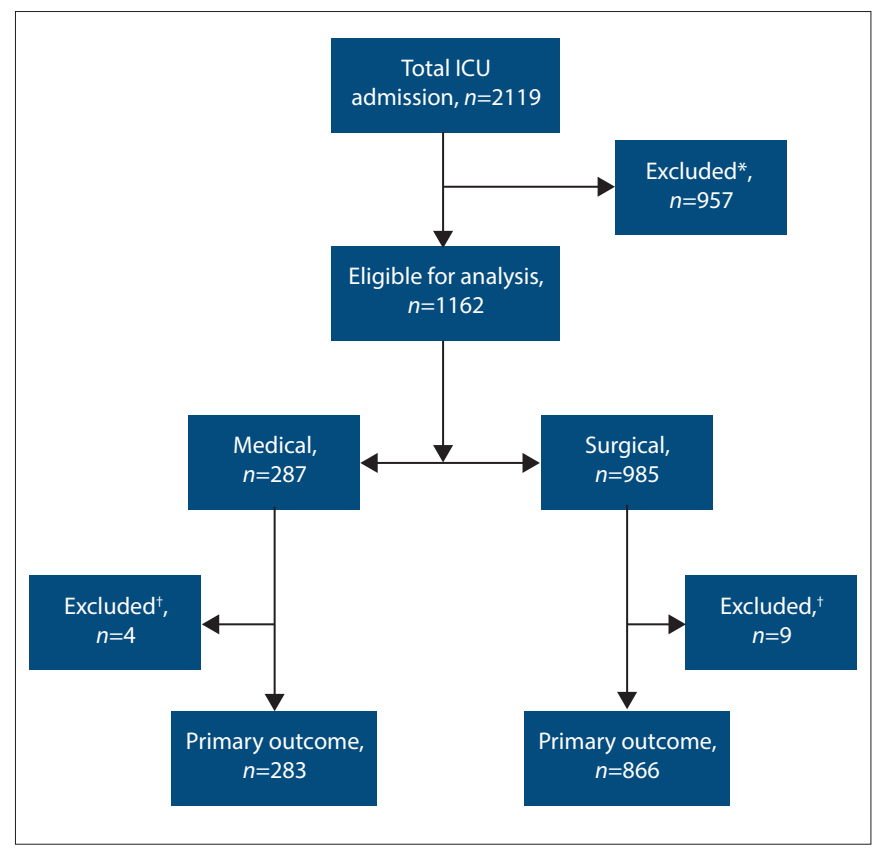

Fig. 1. Consort diagram. $\left({ }^{*}\right.$ No suspected infection, $\mathrm{n}=929 ;$ unknown referring speciality, $\mathrm{n}=28$.) ( ${ }^{\dagger}$ Missing data, $\mathrm{n}=13$.) 
using the $\chi^{2}$ test. All analyses were stratified by medical $\mathrm{v}$. surgical patients.

For all analyses, a $p<0.05$ was considered to be statistically significant, and the precision of estimates was reported with $95 \%$ confidence interval (CI).

\section{Ethics and consent}

Ethics (ref. no. BREC/00001595/2020) and ICU database (ref. no. BCA 211/14) approvals were granted by the University of KwaZulu-Natal Biomedical Research Ethics Committee (BREC). The study protocol was also approved by the Institutional Review Board of the University of Pennsylvania (Philadelphia, USA).

\section{Results}

A total of 2119 adults were admitted to the ICU and screened for eligibility during the

Table 1. Patient characteristics

\begin{tabular}{|c|c|c|}
\hline & $\begin{array}{l}\text { Medical }(n=287) \\
n(\%)^{*}\end{array}$ & $\begin{array}{l}\text { Surgical }(n=875), \\
n(\%)^{*}\end{array}$ \\
\hline Age (years), mean (SD) & $41.51(15.59)$ & $40.39(15.97)$ \\
\hline Female & $145(50.5)$ & $410(46.9)$ \\
\hline Pre-ICU hospital LoS (days), median (IQR) & $1(0-1)$ & $1(0-3)$ \\
\hline Mechanically ventilated $^{\dagger}$ & $161(56.1)$ & $533(60.9)$ \\
\hline \multicolumn{3}{|l|}{ HIV status } \\
\hline Negative & $182(63.4)$ & $669(76.5)$ \\
\hline Positive, taking ART & $73(25.4)$ & $165(18.9)$ \\
\hline Positive, not taking ART & $32(11.2)$ & $41(4.7)$ \\
\hline Maximal respiratory rate, mean (SD) & $31(15)$ & $27(14)$ \\
\hline $\begin{array}{l}\text { Minimum systolic blood pressure ( } \mathrm{mmHg} \text { ), } \\
\text { mean (SD) }\end{array}$ & $100(20)$ & $102(20)$ \\
\hline Glasgow Coma Score, mean (SD) & $9(5)$ & $8(5)$ \\
\hline \multicolumn{3}{|l|}{ qSOFA scores } \\
\hline $0-1$ & $79(27.5)$ & $325(37.1)$ \\
\hline 2 & $127(44.2)$ & $368(42.1)$ \\
\hline 3 & $77(26.8)$ & $176(20.1)$ \\
\hline Missing & $4(1.4)$ & $6(0.7)$ \\
\hline Deaths & $72(25.1)$ & $148(16.9)$ \\
\hline \multicolumn{3}{|c|}{$\begin{array}{l}\text { ART = antiretroviral therapy; ICU = intensive care unit; IQR = interquartile range; LoS = length of stay; } \\
\text { qSOFA = Quick Sepsis-related Organ Failure Assessment; } S D=\text { standard deviation. } \\
\text { *Unless otherwise specified. } \\
\text { tAt time of ICU admission. }\end{array}$} \\
\hline
\end{tabular}

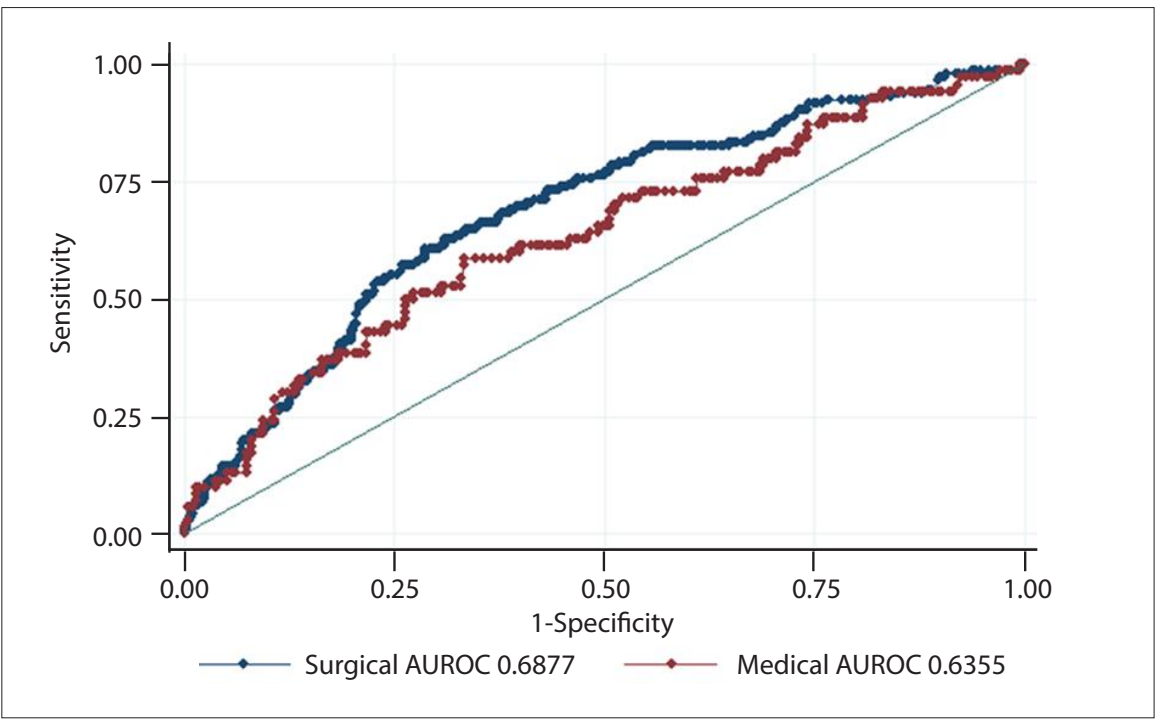

Fig. 2. qSOFA area under the receiver operating characteristic curve (AUROC) for medical v. surgical multivariable logistic regression models. study period. A total of 1162 participants (surgical $n=875$ and medical $n=287$ ) with infection were included in the analysis (Fig. 1). Patient characteristics are described in Table 1.

The number of accepted patient referrals from the surgical disciplines were as follows: general surgery $(54.1 \%$; $n=473)$, trauma (25.5\%; $n=223)$, orthopaedics $(6.9 \% ; n=60)$, obstetrics $(6.4 \% ; n=56)$, gynaecology $(4.0 \%$; $n=35)$, burns $(1.6 \% ; n=14)$, anaesthesia $(0.6 \%$; $n=5)$, urology $(0.6 \% ; n=5)$, otolaryngology $(0.3 \% ; n=3)$, and maxillofacial $(0.1 \% ; n=1)$.

Univariate and adjusted multivariate models demonstrating the associations between qSOFA and age, sex and HIV status with in-ICU mortality are shown in Table 2. In both the unadjusted and adjusted models for medical (unadjusted odds ratio (OR) 2.85; 95\% confidence interval (CI) $1.31-6.18$; $p=0.01$; adjusted OR 2.60; 95\% CI 1.19 - 5.71; $p=0.02$ ) and surgical (unadjusted OR 2.88; 95\% CI 1.83 - 4.54; $p<0.001$; adjusted OR 2.74; $95 \%$ CI 1.73 - 4.36; $p<0.001$ ) subgroups, a qSOFA score of 3 but not a qSOFA score of 2 , was associated with increased odds of inICU mortality compared with a qSOFA score of 0 - 1 .

The AUROCs for the baseline model, qSOFA alone, and qSOFA with the baseline model were all poor ( $95 \%$ CI 0.59 - 0.69) and there were no differences between performance in the medical and surgical subgroups (all $p \geq 0.26$ ), but addition of qSOFA to the baseline model improved performance in both subgroups (both $p<0.05$ ) (Table 3 , Fig. 2 and appendix Figs 1 and 2). Sensitivity analyses with qSOFA treated as a dichotomous score $(<2 \mathrm{v}$. $\geq 2)$ showed similar but attenuated relationships and performance (appendix Tables 1 and 2 (https://www.samedical.org/ file/1677) and appendix Figs 3 and 4 (https:// www.samedical.org/file/1678)). When restricting to patients for whom infection was the primary ICU admission diagnosis ( $n=428$; surgical $n=276$ and medical $n=152$ ), the results were similar (eTables 3 and 4 ) but with some loss of statistical significance in the setting of a smaller sample size and reduced power.

\section{Discussion}

Our study demonstrates that a higher qSOFA score at the time of ICU referral is associated with higher in-ICU mortality in patients with suspected infection in both medical and 
Table 2. Association of qSOFA and baseline characteristics with in-ICU mortality among medical and surgical patients with infection

\begin{tabular}{|c|c|c|c|c|}
\hline & $\begin{array}{l}\text { Medical, } \\
\text { OR }(95 \% \text { CI })\end{array}$ & $p$-value & $\begin{array}{l}\text { Surgical, } \\
\text { OR }(95 \% \mathrm{CI})\end{array}$ & $p$-value \\
\hline \multicolumn{5}{|l|}{ Univariate } \\
\hline \multicolumn{5}{|l|}{ qSOFA (ref: 0 - 1) } \\
\hline $\mathrm{qSOFA}=2$ & $1.88(0.90-3.92)$ & 0.09 & $0.98(0.63-1.52)$ & 0.93 \\
\hline $\mathrm{qSOFA}=3$ & $2.85(1.31-6.18)$ & 0.01 & $2.88(1.83-4.54)$ & $<0.001$ \\
\hline Age (per 10 years) & $0.92(0.77-1.10)$ & 0.34 & $1.23(1.16-1.43)$ & $<0.001$ \\
\hline Male & $0.73(0.42-1.26)$ & 0.26 & $0.55(0.39-0.80)$ & 0.001 \\
\hline HIV-positive & $1.78(1.03-3.09)$ & 0.04 & $1.10(0.72-1.66)$ & 0.67 \\
\hline \multicolumn{5}{|l|}{ Adjusted } \\
\hline \multicolumn{5}{|l|}{ qSOFA (ref: 0 - 1) } \\
\hline $\mathrm{qSOFA}=2$ & $1.81(0.86-3.79)$ & 0.12 & $0.93(0.59-1.45)$ & 0.74 \\
\hline $\mathrm{qSOFA}=3$ & $2.60(1.19-5.71)$ & 0.02 & $2.74(1.73-4.36)$ & $<0.001$ \\
\hline Age (per 10 years) & $1.00(0.98-1.01)$ & 0.63 & $1.02(1.01-1.04)$ & $<0.001$ \\
\hline Male & $0.80(0.46-1.39)$ & 0.43 & $0.62(0.43-0.92)$ & 0.02 \\
\hline HIV-positive & $1.56(0.87-2.78)$ & 0.14 & $1.01(0.65-1.57)$ & 0.97 \\
\hline
\end{tabular}

Table 3. Discrimination of qSOFA score and baseline risk model for in-ICU mortality among medical and surgical patients with infection

\begin{tabular}{|c|c|c|c|}
\hline & $\begin{array}{l}\text { Medical, } \\
\text { AUROC (95\% CI) }\end{array}$ & $\begin{array}{l}\text { Surgical, } \\
\text { AUROC (95\% CI) }\end{array}$ & $\begin{array}{l}\text { Medical v. surgical, } \\
p \text {-value }\end{array}$ \\
\hline qSOFA alone & $0.60(0.53-0.67)$ & $0.60(0.55-0.65)$ & 0.32 \\
\hline Baseline model ${ }^{*}$ & $0.59(0.51-0.67)$ & $0.64(0.59-0.69)$ & 0.94 \\
\hline \multirow{2}{*}{$\begin{array}{l}\text { qSOFA + baseline } \\
\text { model }\end{array}$} & $0.64(0.56-0.71)$ & $0.69(0.64-0.74)$ & 0.26 \\
\hline & $p$-value & & \\
\hline $\begin{array}{l}\text { Addition of qSOFA } \\
\text { to baseline model }\end{array}$ & 0.049 & $<0.0001$ & - \\
\hline \multicolumn{4}{|c|}{$\begin{array}{l}\text { qSOFA = quick Sequential Organ Failure Assessment; } \mathrm{ICU}=\text { intensive care unit; } \mathrm{AUROC}=\text { area under the receiver operating } \\
\text { characteristic curve; } \mathrm{CI}=\text { confidence interval. }\end{array}$} \\
\hline
\end{tabular}

surgical patient populations in a resourcelimited setting, with no statistically significant differences between groups.

The addition of qSOFA at the time of ICU referral to a baseline model known to predict in-ICU mortality (age, sex, HIV status) improved the performance of the model in both surgical and medical patients, but discrimination of all qSOFA models dichotomous or ordinal, with and without baseline factors was poor. The use of a baseline model allows for assessment of the incremental benefit of adding qSOFA to clinical factors that are familiar to clinicians rather than a complex multivariable model.

Risk scores have traditionally been used to measure disease severity and quantify the risk of death in an ICU population rather than in an individual patient. ${ }^{[20]}$ This allows for comparisons between clinical trials, and to benchmark quality of care between ICUs. Commonly used scores such as the Acute Physiologic and Chronic Health Evaluation $(\mathrm{APACHE})^{[21]}$ and the Simplified Acute Physiologic Score (SAPS) ${ }^{[22]}$ are widely used, regularly updated, and incorporate a number of physiological variables taken within the first 24 hours following ICU admission. However, they are not easily operationalised for bedside use, especially in resource-limited settings due to the large number of inputs and requirement for laboratory-based testing, and because they incorporate some variables collected following ICU admission rather than just at admission.

Early warning systems (EWS) such as the Modified Early Warning Score (MEWS) and the National Early Warning Score (NEWS) have been developed to identify patients at risk of deterioration. These scores are also widely used and perform well in comparison to qSOFA in the prediction of in-hospital mortality and ICU transfer ${ }^{[23]}$ However, they are also more complex than qSOFA, and therefore are less suitable as a simple, bedside assessment tool. The Sequential (sepsisrelated) Organ Failure Assessment (SOFA) score was initially designed to assess the degree of severity of organ dysfunction in septic patients. ${ }^{[24]}$ The qSOFA score was subsequently proposed as a means to identify patients with suspected infection at risk for poor outcomes and was first validated outside of the ICU setting. ${ }^{[2,25]}$ The early recognition of sepsis and patients with suspected infection at risk for poor outcomes is increasingly recognised as a key area of possible intervention. ${ }^{[2]}$

Our findings are largely consistent with recent emerging studies on qSOFA in resource-limited settings. ${ }^{[13,18]}$ In total, these findings suggest that qSOFA can be extended from its initial validation population of nonICU patients with infection in well-resourced settings to patients in ICUs in resource-limited settings, both with and without infection, and both medical and surgical varieties of critical illness. While qSOFA was originally developed and validated in primarily medical populations, the qSOFA components carry similar prognostic weights in both medical and surgical patients. As discrimination remains poor, qSOFA should remain one tool in a clinician's kit to be used in context with other risk assessment and prognostic approaches. Further prospective evaluation of the role of qSOFA in these settings is advised.

\section{Study strengths and limitations}

Our study has several limitations, including its single-centre, retrospective design. Results should not be generalised unless as part of the totality of emerging evidence from multiple sites globally. Specifically, the study ICU may have lower mortality than regional peers. Our ICU is also intensivist-led, staffed with on-site registrars, and as such may represent a better-resourced environment than many lower-income settings. Finally, in the primary analysis we defined infection in any patient in whom antibiotics had been initiated at, or prior to, admission to ICU based on expert local opinion on antibiotic prescribing patterns in the study hospital and ICU. One study suggested that inappropriate empiric antibiotics were prescribed in over half of ICU admissions in SA. ${ }^{[2]}$ This is mitigated by 
a strict antibiotic stewardship policy in the study ICU. But this approach may have omitted patients with undetected infection and included patients prescribed antibiotics for indications other than acute infection (e.g. prophylaxis, chronic infection). This was addressed in part by examining a highly specific subgroup of patients for whom infection was the primary ICU admission diagnosis with overall similar results.

Our study has several strengths. It is the first to our knowledge that aims to directly compare the use of qSOFA in medical and surgical patients. This may be an important differentiator given the different pathophysiological origins of infection in these populations.

A previous study compared the qSOFA with the SIRS score in a mixed population of medical and surgical patients, and showed that qSOFA had better prognostic value (AUROC $0.86 \mathrm{v} .0 .67$ ) for poorer outcomes. ${ }^{[27]}$ However, there was no direct comparison to examine its utility in medical v. surgical patients.

Secondly, it supported the potential utility of qSOFA in a resourcelimited setting where the more extensive laboratory work-up required for a full SOFA score calculation may not be immediately available. The qSOFA score uses only blood pressure, respiratory rate, and mentation. These parameters are available in nearly any setting using bedside assessment alone. Routinely calculating the qSOFA score when assessing a patient for possible ICU referral as a standard of care may increase experience with the score, without adding to the clinical burden. Our study specifically used qSOFA scores taken prior to in-ICU interventions.

\section{Conclusion}

A higher qSOFA score was associated with higher in-ICU mortality in medical and surgical patients with suspected infection admitted to ICU in a resource-limited setting in SA. These findings suggest that qSOFA may be useful as one tool or as an adjunct to other risk assessment and prognostic approaches to identify patients most at risk of poor outcomes.

\section{Declaration. None.}

Acknowledgements. We would like to acknowledge Dr Nikki Allorto for her work in design and maintenance of the intensive care electronic database (ICED) used in this study. We also acknowledge Dr Carel Cairns for his prior work on data captured on the ICED system.

Author contributions. LAB, DW, RDW, SMS and GLA conceptualised and designed the study. LAB analysed and interpreted the data and wrote the manuscript. GLA performed primary statistical analysis and interpretation of data. DW, RDW and SMS revised the manuscript. All authors approved the manuscript for submission.

Funding. This project was supported by U.S. Agency for Healthcare Research and Quality (K12HS026372) for GLA.

Conflicts of interest. GLA reports receiving fees from UpToDate and for expert witness consulting.

1. Vincent J-L, Marshall JC, Namendys-Silva SA, et al. Assessment of the worldwide burden of critical illness: The Intensive Care Over Nations (ICON) audit. Lancet Resp Med 2014;2(5):380-386. https://doi.org/10.1016/s2213-2600(14)70061-x

2. Seymour CW, Liu VX, Iwashyna TJ, et al. Assessment of clinical criteria for sepsis: For the third international consensus definitions for sepsis and septic shock (Sepsis-3). JAMA 2016;315(8):762-774. https://doi:10.1001/jama.2016.0288.

3. Anesi GL, Gabler NB, Allorto NL, et al. Intensive care unit capacity strain and outcomes of critical illness in a resource-limited setting: A 2-hospital study in South Africa. J Intensive Care Med 2018:885066618815804. https://doi.org/10.1177/0885066618815804
4. Napolitano LM. Sepsis 2018: Definitions and guideline changes. Surg Infect (Larchmt) 2018;19(12):117-125. https://doi.org/10.1089/sur.2017.278

5. Fernando SM, Tran A, Taljaard M, et al. Prognostic accuracy of the quick sequential organ failure assessment for mortality in patients with suspected infection: A systematic review and meta-analysis. Ann Intern Med 2018;168(4):266-275. https:// doi.org/10.7326/M17-2820

6. Liu YC, Luo YY, Zhang X, et al. Quick sequential organ failure assessment as a prognostic factor for infected patients outside the intensive care unit: A systematic review and metaanalysis. Intern Emerg Med 2019;14(4):603-615. https://doi.org/10.1007/s11739-01902036-0

7. Lo RSL, Leung LY, Brabrand M, et al. qSOFA is a poor predictor of short-term mortality in all patients: A systematic review of 410000 patients. J Clin Med 2019;8(1):61. https:// doi.org/10.3390/jcm8010061

8. Maitra S, Som A, Bhattacharjee S. Accuracy of quick sequential organ failure assessment (qSOFA) score and systemic inflammatory response syndrome (SIRS) criteria for predicting mortality in hospitalised patients with suspected infection: A meta-analysis of observational studies. Clin Microbiol Infect 2018;249(11):1123-1129. https://doi. org/10.1016/j.cmi.2018.03.032

9. Raith EP, Udy AA, Bailey M, et al. Prognostic accuracy of the SOFA score, SIRS criteria, and qSOFA score for in-hospital mortality among adults with suspected infection admitted to the intensive care unit. JAMA 2017;317(3):290-300. https://doi.org/10.1001/ jama.2016.20328

10. Serafim R, Gomes JA, Salluh J, Povoa P. A comparison of the quick-SOFA and systemic inflammatory response syndrome criteria for the diagnosis of sepsis and prediction of mortality: A systematic review and meta-analysis. Chest 2018;153(3):646-655. https:// doi.org/10.1016/j.chest.2017.12.015

11. Song JU, Sin CK, Park HK, Lee J. Performance of the quick sequential (sepsis-related) organ failure assessment score as a prognostic tool in infected patients outside the intensive care unit: A systematic review and meta-analysis. Crit Care 2018;22(1):28. https://doi.org/10.1186/s13054-018-1952-x

12. Tan TL, Tang YJ, Ching LJ, Abdullah N, Neoh HM. Comparison of prognostic accuracy of the quick sepsis-related organ failure assessment between short- and longterm mortality in patients presenting outside of the intensive care unit - a systematic review and meta-analysis. Sci Rep 2018;8(1):16698. https://doi.org/10.1038/s41598018-35144-6

13. Rudd KE, Seymour CW, Aluisio AR, et al. Association of the quick sequential (sepsisrelated) organ failure assessment (qSOFA) score with excess hospital mortality in adults with suspected infection in low- and middle-income countries. JAMA 2018;319(12):2202-2211. https://doi.org/10.1001/jama.2018.6229

14. Aluisio AR, Garbern S, Wiskel T, et al. Mortality outcomes based on ED qSOFA score and HIV status in a developing country. Am J Emerg Med 2018;36(11):2010-2019. https://doi.org/10.1016/j.ajem.2018.03.014

15. Boillat-Blanco N, Mbarack Z, Samaka J, et al. Prognostic value of quickSOFA as a predictor of 28-day mortality among febrile adult patients presenting to emergency departments in Dar es Salaam, Tanzania. PLoS ONE 2018;13(6):e0197982. https://doi. org/10.1371/journal.pone.0197982

16. Carugati M, Zhang HL, Kilonzo KG, et al. Predicting mortality for adolescent and adult patients with fever in resource-limited settings. Am J Trop Med Hyg 2018;99(5):12461254. https://doi.org/10.4269/ajtmh.17-0682

17. Huson MA, Kalkman R, Grobusch MP, van der Poll T. Predictive value of the qSOFA score in patients with suspected infection in a resource-limited setting in Gabon. Travel Med Infect Dis 2017;15:76-77. https://doi.org/10.1016/j.tmaid.2016.10.014

18. Savarimuthu SM, Cairns C, Allorto NL, et al. qSOFA as a predictor of ICU outcomes in a resource-limited setting in South Africa. South Afr J Crit Care 2020;36(2):92-95. https:// doi.org/10.7196/SAJCC.2020.v36i2.433

19. Allorto NL, Wise RD. Development and evaluation of an integrated electronic data management system in a South African metropolitan critical care service. South Afr J Anaesth Analg 2015;21(6):31-35. https://doi:10.1080/22201181.2015.1115607

20. Vincent JL, Moreno R. Clinical review: Scoring systems in the critically ill. Crit Care 2010;14(2):207. https://doi.org/10.1186/cc8204

21. Knaus WA, Draper EA, Wagner DP, Zimmerman JE. APACHE II: A severity of disease classification system. Crit Care Med 1985;13(10):818-829.

22. Moreno RP, Metnitz PG, Almeida E, et al. SAPS 3 - from evaluation of the patient to evaluation of the intensive care unit. Part 2: Development of a prognostic model for hospital mortality at ICU admission. Intensive Care Med 2005;31(10):1345-1355. https://doi.org/10.1007/s00134-005-2763-5

23. Churpek MM, Snyder A, Han X, et al. Quick sepsis-related organ failure assessment, systemic inflammatory response syndrome, and early warning scores for detecting clinical deterioration in infected patients outside the intensive care unit. Am J Respir Crit Care Med 2017;195(7):906-911. https://doi.org/10.1164/rccm.201604-0854OC

24. Vincent JL, de Mendonca A, Cantraine F, et al. Use of the SOFA score to assess the incidence of organ dysfunction/failure in intensive care units: Results of a multicenter, prospective study. Working group on 'sepsis-related problems' of the European Society of Intensive Care Medicine. Crit Care Med 1998;26(11):1793-1800. https://doi. org/10.1097/00003246-199811000-00016 
25. Singer M, Deutschman CS, Seymour CW, et al. The third international consensus definitions for sepsis and septic shock (Sepsis-3). JAMA 2016;315(8):801-810. https:/ doi.org/10.1001/jama.2016.0287

26. Paruk F, Richards G, Scribante J, Bhagwanjee S, Mer M, Perrie H. Antibiotic prescription practices and their relationship to outcome in South Africa: Findings of the prevalence of infection in South African intensive care units (PISA) study. S Afr Med J 2012;102(7):613-616. https://doi.org/10.7196/samj.5833
27. Luo J, Jiang W, Weng L, et al. Usefulness of qSOFA and SIRS scores for detection of incipient sepsis in general ward patients: A prospective cohort study. J Crit Care 2019;51:13-18. https://doi.org/10.1016/j.jcrc.2019.01.012

Accepted 27 September 2021. 